\title{
THE
}

\section{Proportional fitness loss and the timing of defensive investment: a cohesive framework across animals and plants}

\author{
Michael J. Sheriff \\ John L. Orrock \\ Maud CO Ferrari \\ Richard Karban \\ Evan L. Preisser \\ University of Rhode Island, preisser@uri.edu
}

See next page for additional authors

Follow this and additional works at: https://digitalcommons.uri.edu/bio_facpubs

The University of Rhode Island Faculty have made this article openly available.

Please let us know how Open Access to this research benefits you.

This is a pre-publication author manuscript of the final, published article.

Terms of Use

This article is made available under the terms and conditions applicable towards Open Access Policy Articles, as set forth in our Terms of Use.

\section{Citation/Publisher Attribution}

Sheriff, M.J., Orrock, J.L., Ferrari, M.C.O. et al. Proportional fitness loss and the timing of defensive investment: a cohesive framework across animals and plants. Oecologia 193, 273-283 (2020).

https://doi.org/10.1007/s00442-020-04681-1 
Authors

Michael J. Sheriff, John L. Orrock, Maud CO Ferrari, Richard Karban, Evan L. Preisser, Andrew Sih, and Jennifer S. Thaler

This article is available at DigitalCommons@URI: https://digitalcommons.uri.edu/bio_facpubs/235 


\section{Proportional fitness loss and the timing of defensive investment: a}

\section{cohesive framework across animals and plants}

4 Running Title: PFL and the timing of defensive investment

6 Michael J. Sheriff ${ }^{*}$, John L. Orrock ${ }^{2 \dagger}$, Maud C. O. Ferrari ${ }^{3}$, Richard Karban ${ }^{4}$, Evan L. Preisser ${ }^{5}$, 7 Andrew $\mathrm{Sih}^{6}$, Jennifer S. Thaler ${ }^{7}$

$9{ }^{1}$ Biology Department, University of Massachusetts Dartmouth, Dartmouth, MA, USA

$10 \quad{ }^{2}$ Department of Integrative Biology, University of Wisconsin, Madison, WI, USA

$11{ }^{3}$ Department of Biomedical Sciences, WCVM, University of Saskatchewan, Saskatoon, SK, 12 Canada

$13{ }^{4}$ Department of Entomology, University of California, Davis, CA, USA

$14{ }^{5}$ Department of Biological Sciences, University of Rhode Island, Kingston, RI, USA

$15{ }^{6}$ Department of Environmental Science and Policy, University of California, Davis, CA, USA

$16{ }^{7}$ Department of Entomology, Cornell University, Ithaca, NY, USA

18 †Subsequent coauthors listed alphabetically

19 *Correspondence:

20 Michael J Sheriff

21 msheriff@umassd.edu

24 Declaration of Authorship All authors conceived of the concepts and ideas. MJS led the writing

25 with significant contributions from all others. 


\section{Abstract}

27 The risk of consumption is a pervasive aspect of ecology and recent work has focused on

28 synthesis of consumer-resource interactions (e.g., enemy-victim ecology). Despite this, theories

29 pertaining to the timing and magnitude of defenses in animals and plants have largely developed

30 independently. However, both animals and plants share the common dilemma of uncertainty of

31 attack, can gather information from the environment to predict future attacks and alter their

32 defensive investment accordingly. Here, we present a novel, unifying framework based on the

33 way an organism's ability to defend itself during an attack can shape their pre-attack investment

34 in defense. This framework provides a useful perspective on the nature of information use and

35 variation in defensive investment across the sequence of attack-related events, both within and

36 among species. It predicts that organisms with greater proportional fitness loss if attacked will

37 gather and respond to risk information earlier in the attack sequence, while those that have lower

38 proportional fitness loss may wait until attack is underway. This framework offers a common

39 platform to compare and discuss consumer effects and provides novel insights into the way risk

40 information can propagate through populations, communities, and ecosystems.

46 Keywords: predation risk, herbivory, induced defense, anti-predator response, information, non47 consumptive effects, trait-mediated effects, vulnerability 


\section{Animals and plants: both must cope with consumers}

50 Consumption is a pervasive feature of ecological systems, yet our understanding of predator-prey

51 and plant-herbivore interactions has largely developed independently. This separation may have

52 its origins in influential historical work arguing that while prey were limited by predators and

53 parasites, plants were limited by resources rather than their consumers (e.g., Hairston et al.

54 1960). More recently, ecologists have begun to appreciate the similarities among consumers'

55 effects, particularly parasite and predator effects on prey; e.g., enemy-victim ecology (Lafferty

56 and Kuris 2002; Raffel et al. 2008; Lafferty et al. 2015). Far fewer studies have examined the

57 commonality of prey and plant responses to their respective consumers (e.g., Hunter 2016;

58 Karban et al. 2016; Niu et al. 2018). The separate developmental trajectories of these disciplines

59 reflect their obvious differences. Many prey, for example, can employ an array of cognitive and

60 behavioral adaptations to detect predators, track risk in the environment, and avoid predation,

61 because any successful attack is likely to prove lethal. In contrast, plants are rooted in place,

62 have historically been thought to lack sophisticated sensory abilities and complex neural

63 architecture, and can often survive partial consumption. Despite the differences in predator-prey

64 and plant-herbivore interactions, both systems share a common dilemma: when to invest in

65 defense against attack. While considerable attention has been given to understanding the

66 substantial intra- and interspecific variation in defensive investment (e.g., Coley et al. 1985;

67 Lima and Bednekoff 1999; Sih 1992; Stamp 2003; Orrock et al. 2015), differences across taxa

68 are often difficult to reconcile. We suggest that a simple framework based on characteristics that

69 prey and plants share, i.e., differences in proportional fitness loss across the sequence of attack-

70 related events and the ability to gather and use information about the probability of attack, can

71 add to our understanding of predator-prey and herbivore-plant interactions and provide insight 
72 into the variation in defense of both prey and plants.

73 Prey and plants must balance the costs of being consumed (or damaged) with the costs of

74 unnecessary defense. They share a common solution to this problem: when information is not too

75 costly to gather (Sih 1992; Chittka et al. 2009), both prey and plants use environmental cues to

76 fine-tune their defensive strategies (Karban et al. 1999; Stankowich and Blumstein 2005). This is

77 one of the foundations of the 'ecology of fear', whose implications have been well studied in

78 predator-prey systems (Brown et al. 1999; Clinchy et al. 2013; Sheriff et al. 2020). Plants can

79 also adjust their responses to both the timing and nature of cues about herbivory (Karban and

80 Baldwin 1997; Karban et al. 1999; Heil 2014), with consequences extending beyond the focal

81 plant (Ohgushi 2005). Given the ubiquity of defensive plasticity and widespread use of

82 information to tailor defensive investment in both prey and plants (Caro 2005; Karban et al.

83 2016), we suggest that an organism's proportional fitness loss if attacked (PFL; Box 1), rather

84 than the prey/plant classification per se, most strongly influences the nature of information

85 gathering and use.

86 We propose a framework for predicting the dynamics of defensive investment based upon

87 the rate at which expected fitness is lost across the sequence of attack-related events of predator-

88 prey or herbivore-plant interactions (Fig. 1). Importantly, defensive investment across the

89 sequence depends upon both the costs of not responding and the costs of responding too early,

90 both of which can depend not only on an individual's PFL and their willingness to pay such

91 costs, but also on individual state (e.g., risk of starvation; Box 1). The idea that an individual's

92 PFL is a key factor determining the timing and magnitude of the response to risk of consumption

93 has several implications. First, it provides a unifying, common platform among taxa to discuss

94 and compare variable and often context-specific responses to the risk of predation or herbivory. 
95 In doing so, it provides a predictive framework for understanding which individuals within a

96 population and which species within a community will be most responsive to changes in the risk

97 of consumption. For example, it provides a clear explanation of why predation risk appears to

98 evoke stronger reactions in small mammals like mice than large ungulates like elk. Our

99 framework reveals that mice have a far higher potential PFL and thus respond earlier - but not

100 necessarily 'more' - than elk (Fig. 1), which may simply be delaying their response given their

101 low PFL if attacked (thus the timing of when prey responses are recorded may significantly

102 biased our perspective of risk responses). Second, it provides novel insights into how

103 information about risk can propagate through populations, communities, and ecosystems

104 depending upon the comparative PFL of species within a given system.

105

106

\section{Defensive investment across a common interaction sequence}

107 The concept of PFL requires an appreciation for the common sequence of attack-related

108 events shared during both predator-prey and herbivore-plant interactions (pre-encounter

109 spatiotemporal overlap, encounter, detection, attack, capture, consumption, post-interaction

110 escape/recovery; Lima and Dill 1990; Karban and Baldwin 1997; Caro 2005; Guiden et al. 2019;

111 Fig. 2). Both prey and plants can alter the outcome of interactions with their attackers by altering

112 the timing of defensive investment as the sequence proceeds.

113 Pre-encounter Spatiotemporal Overlap. In consumer-resource interactions, the first step in the

114 interaction sequence is spatiotemporal overlap - when consumers and resources occupy the same

115 area at the same time (Schmitz et al. 2017). Consumers should generally seek to increase this

116 overlap, while their resources should attempt to reduce it (i.e., the space-race concept; Sih 2005);

117 mobile prey have a great advantage in this compared to plants. Defensive initiation at this stage 
118 will reduce encounter probability but may come at the costs of unnecessary defense (e.g., not

119 occupying an area of high food availability). Importantly, defensive investment at this stage

120 depends upon information gained by the prey or plant during a prior predation/herbivory event

121 and the potential PFL of the individual based upon this prior event.

122 Encounter. An encounter occurs when the distance between a predator and prey is less than the

123 detection range of one or both participants (Lima and Dill 1990). This definition of encounter

124 also applies to herbivore-plant systems; although herbivores typically detect plants from greater

125 distances, plants can detect cues, including volatile signals from other plants and herbivores

126 (Helms et al. 2017, 2019; Markovic et a. 2019). While spatial overlap has traditionally been

127 used to evaluate encounter probability, recent studies also emphasize the importance of temporal

128 overlap (Guiden et al. 2019). Prey can thus avoid encounters by reducing their use of risky areas

129 in both space (e.g., landscape of fear, Laundré et al. 2001) and time (e.g., Smith et al. 2019).

130 Although plants are less able to avoid spatial overlap with herbivores, they may alter their

131 defensive investment in time to avoid encounters and reduce the probability of herbivore attack.

132 For example, foliar nyctinasty (daily movement of plant leaves) may reduce encounters with

133 herbivores by reducing leaf availability at night (Minorsky 2019). There is also evidence that

134 plants may preempt attack in time by adjusting their defensive investment to be greater during

135 times of day when encounter with an attacker is more likely (Falk et al. 2014).

136 Detection. Detection can occur sequentially (e.g., the consumer first detects the prey or plant, or

137 vice versa) or simultaneously (the consumer and victim detect each other at the same time).

138 Defensive initiation at this stage requires prey and plants to gather, identify (e.g., recognize the

139 consumer as a threat), and respond to risk information. The type and magnitude of defensive

140 initiation will depend upon costs and benefits of a particular response given the information 
141 provided by the cue (Orrock et al. 2015). For example, cues indicative of imminent attack (e.g.,

142 auditory cues or consumption of conspecifics) are likely to evoke greater responses than cues

143 (e.g., feces or urine) that only suggest consumers are within the vicinity (Kim et al. 2011;

144 Hermann and Thaler 2014; Parsons et al. 2017). It is important to note, however, that even

145 informed resources (be they prey or plants) may not respond as expected due to other constraints

146 (McNamara and Houston 1986; Brown and Kotler 2004; Sheriff et al. 2020). For example,

147 Nucella lapillus snails (prey) adjusted their foraging response to predatory crabs relative to their

148 body condition (Matassa et al. 2016). Some plants will modify their touch-induced leaf-closing

149 time as a function of available resources (Jensen et al. 2011).

150 These responses can be considered within our framework and will depend upon the PFL

151 of the prey or plant if attack occurs. For example, state-dependent foraging theory (McNamara

152 and Houston 1992) predicts organisms in good condition should initiate defenses early because

153 they can pay the cost of reduced foraging. This dovetails with predictions from our framework:

154 good-condition individuals also have the highest initial fitness potential and thus a relatively high

155 PFL if attacked. In contrast, poor-condition individuals have relatively low initial fitness

156 potential (i.e., they may die regardless of risk) and thus a lower PFL if attacked (Box 1).

157 Attack, Capture, and Consumption. If consumers detect prey or plants an attack may occur (e.g.,

158 an approach or chase), which may result in the initiation of consumption if the prey or plant is

159 incapable of avoiding capture. It is at these stages that a clear distinction arises between prey and

160 plants. First, capture is less likely for mobile prey than for immobile plants. Further, once an

161 attack is initiated, prey must initiate defense to avoid their likely-lethal capture and consumption.

162 Plants, particularly mature individuals, may take advantage of the potentially low PFL to wait

163 until attack or even consumption begins to initiate defense (Karban and Baldwin 1997; Kim et al. 
164 2011). Interestingly, this does not necessarily hold true for seed predation, which is often lethal

165 to immature plants; consistent with our PFL framework, seeds are often highly defended prior to

166 any attack. Within the idea of PFL, the initiation of defense prior to attack and consumption will

167 depend upon the ability of organisms to defend during an attack (Box 1).

168 Post-interaction Escape/Recovery. At any point in the sequence prey and plants may end the

169 interaction depending upon the timing of their defensive investment and ability to evade their

170 consumer. Because consumption is generally lethal for prey, this would benefit prey most prior

171 to their capture; for plants, this is likely to occur post-consumption. Importantly, this stage is not

172 the end of the defensive investment for individuals. For example, we expect individuals with

173 higher PFL to prolong their post-interaction defensive investment relative to individuals with

174 lower PFL (Sih 1992; Gil et al. 2018). This past experience will also prime individuals for their

175 next encounter; their PFL will likely alter the timing and magnitude of their defensive investment

176 during the pre-encounter spatiotemporal overlap stage.

177

178 A unifying framework to understand defensive investment among taxa

179 Proportional fitness loss as an underlying principle

180 The concept of PFL, as we define it here (Box 1), can be characterized broadly across

181 taxa as the proportional loss of relative fitness if an individual does not initiate defense until

182 attacked. This proportional loss of relative fitness accounts for both the ability of an individual to

183 defend early in the interaction sequence and its ability to defend during an attack. It can be

184 measured as the fitness potential if defense is initiated prior to attack (e.g., during an encounter

185 or detection) compared to that if defense is initiated after attack has begun (Box 1). As such,

186 these ideas can extend beyond the general prey and plant classification and be used to compare 
187 individuals within and among populations and can also be broadly applied to compare

188 individuals among species.

189 In natural systems, there are several stages along the interaction sequence prior to attack

190 and the beginning of consumption where individuals can initiate defense. For example, ungulates

191 can initiate defense during both the encounter (e.g., alter temporal habitat use) and the detection

192 stage (e.g., fleeing), yet, there are also many cases where predators are able to initiate an attack

193 (e.g., a chase) prior to prey detection and prey are only able to initiate defense (e.g., flee) after

194 the attack has begun. Plants can also detect the presence of herbivores prior to attack (Helms et

195 al. 2017, 2019; Heil and Karban 2010; Orrock et al. 2018), however, there are also times when

196 plants do not detect herbivore presence until capture (e.g., occupation of a leaf, Pfeiffer et al.

197 2009; ovipositing of herbivore eggs onto leaves, Hilker and Meiner 2006) or even consumption

198 has begun, and thus only initiate defense (e.g., increase alkaloids) thereafter (Kim et al. 2011). It

199 is the comparison of the proportional loss of relative fitness if prey or plants initiate defense at a

200 stage prior to attack vs. if they initiate defense during attack that is the practical measure of PFL

201 (Box 1).

202 It is important to appreciate that the efficacy and timing of any increase in defensive

203 investment may depend upon the unique characteristics of the individual prey or plant and the

204 landscape in which they are encountered. Age, health, the presence of chemically or physically

205 defended parts, sensory ability and cue recognition, relative mobility, size as well as landscape

206 features like refuges can all influence the ability to evade and defend against attack and escape

207 consumption (Stankowich and Blumstein 2005; Preisser and Orrock 2012; Karban et al. 2016;

208 Sheriff et al. 2020). For example, size may reduce an individual's PFL by increasing its ability to

209 evade and thwart an attack (e.g., Luttbeg and Sih 2010; Wishingrad et al. 2014); i.e., if attacked, 
210 larger individuals may have stronger escape potential and reduced fitness-loss, shifting

211 individuals from high PFL (dashed line) to low PFL (solid line) in Fig. 1, as they grow.

212 Alternatively, if protecting offspring increases potential fitness costs during an attack,

213 individuals with offspring may have a higher PFL and respond earlier along the interaction

214 sequence, shifting individuals from low PFL (solid line in Fig. 1) to high PFL (dashed line in

215 Fig. 1).

216 It is also important to remember that prey and plants express defenses at some baseline

217 level (i.e., constitutive defenses) independent of risk cues. Use of constitutive defenses are

218 expected when levels of attack are consistently high, when cues from the environment are not

219 useful in predicting attack, or when defenses cannot be induced quickly enough. Work on

220 Trinidadian guppies has shown that their life-history traits (i.e., antipredator behavior) depend

221 upon whether they live in systems with high vs low predation (Reznick and Endler 1982;

222 Reznick et al. 1990). Importantly, appreciating potential prior exposure to the risk of

223 consumption, whether over evolutionary or ecological time, may alter predictions pertaining to

224 individual PFL and the timing of defensive investment; i.e., prior experience may prime

225 individuals in their defensive response. Within our framework, constitutive defenses can be

226 considered to occur prior to the start of the interaction sequence and thus lower PFL compared to

227 not having constitutive defenses. For example, in many plant species, individuals (or their

228 modular parts) that have a high level of constitutive defense (e.g., high levels of xanthotoxin)

229 display weak defensive investment (e.g., induced increases in xanthotoxin) when attacked

230 compared to those individuals (or parts) that do not display constitutive defenses (Zangerl and

231 Rutledge 1996). Additionally, constitutive defenses could be considered to occur very early

232 given the future potential for an attack-related interaction to occur. For example, many studies in 
233 both prey and plants have shown that parental exposure to predation risk or herbivory results in

234 offspring with greater antipredator/herbivore defenses even though the offspring have yet to

235 experience risk of consumption (Rossiter 1996; Agrawal et al. 1999; Sheriff et al. 2010; Holeski

236 et al. 2012; Sheriff et al. 2017; Tigreros et al. 2017; Donelan et al. 2020). Within our framework

237 we predict that the magnitude of transgenerational or constitutive defensive investment would be

238 greatest in species or populations where naïve individuals (those without prior information,

239 whether transgenerational or evolutionary) have the highest PFL. Thus, we propose that the

240 concept of relative PFL, both within and across taxa, provides a unifying, common framework

241 for determining how and when individuals should gather and utilize information, and underlies

242 an individual's ability to develop and implement defensive strategies for minimizing the fitness

243 costs of an attack.

245 Defense amidst uncertainty

246 Although differences in sensory abilities among taxa must be considered (Karban et al.

247 2016; Weissburg et al. 2014), the timing of when to gather and use risk-related information in the 248 interaction sequence can depend greatly on an individual's PFL (Fig. 1). Since the reliability of 249 risk-related information increases as the sequence progresses, later-responding organisms should 250 be more capable of fine-tuning defensive investment and reduce the costs of unnecessary defense 251 - an advantage that must be balanced against the costs of not responding early enough (Bateman 252 et al. 2014; Orrock et al. 2015). For example, small mammals gather and use information very 253 early in the interaction sequences (e.g., moonlight-induced reductions in activity; Prugh and 254 Golden 2014) and continue throughout the sequence, since captured individuals are unlikely to 255 survive. In fact, many prey will continue to collect and process information even after the 
256

257

258

259

260

261

262

263

264

265

266

267 The transmission and use of risk information

268

269

270

271

272

273

274

275

276

277

278 individuals and communities.

\section{The propagation of risk information among individuals}

encounter ends (likely in preparation for another potential attack), since the ability to track and respond to risk cues even in the absence of an attack is highly beneficial (Sih 1992). At the other end of the spectrum, low- to moderate-levels of herbivory often have such low fitness costs for trees or other large plants (Karban and Baldwin 1997) that they can afford to wait until attack or even consumption to gather and use risk-related information. This concept can be extended beyond the generalized classification of prey or plants; by comparing the relative PFL among individuals or among species within a community predictions can be made within populations and across taxa on the timing and magnitude of defensive investment throughout the interaction sequence (Box 1, Fig. 1). It also has implications for how risk information can propagate among

For many prey, group size and composition are important factors altering the relative risk experienced by a given individual (Hamilton 1971; Bednekoff and Lima 1998a). This may result from improved predator detection (the 'many eyes' hypothesis) or increased dilution of individual risk, assuming that predators can kill only a small number of group members at a time (Bednekoff 1997). Although early models assumed that detection by any group member would provide equal benefits to all members, individuals differ in their vulnerability and information about risk. Within a group, an individual must detect and respond to a predator prior to a certain time point $(t)$ in order to reach safety, or must detect and respond to the primary detector. However, given that a secondary responder has a lag $(l)$ in their response, the primary detector must respond to a predator at $t+l$ in order for the second individual to react in time to reach 
safety (Bednekoff and Lima 1998b). We can extend this model of primary vs. secondary

280 responders to incorporate the concept of PFL. Take, for example, a group in which all

281 individuals have an equivalent fitness potential if they initiate defense very early (e.g., 100\%

282 survival probability). Some group members will have a lower fitness potential if they initiate

283 defense during an attack, and thus, they will have a higher rate of fitness loss across the

284 interaction sequence and a higher PFL (dashed line in Fig 1). Thus, to maintain an equivalent

285 fitness potential, individuals with a higher PFL need to respond earlier in the interaction

286 sequence, and, thus, have a larger value of $t$ (time required to reach safety) compared to

287 individuals with a lower PFL.

288 From this, we can make predictions based on the PFL of the primary, secondary (and

289 tertiary, etc.) responders, which will yield very different outcomes for individual and group

290 responses. Most often, we expect individuals with the highest PFL within a group to be the

291 primary detectors and responders, leaving ample time for other individuals to respond, but this

292 may not always be the case. In semi-fossorial groups (e.g., ground squirrel colonies), individuals

293 living in the periphery of the habitat may have a lower likelihood of surviving an attack, and thus

294 have a higher PFL. However, living in sub-optimal habitats may also lead to reduced visibility

295 (Werner et al. 2015). In such scenarios, individuals with lower PFL may be the primary detectors

296 and, thus, may not respond until later in the interaction sequence, possibly later than the required

297 time $(t+l)$ for a secondary responder, particularly if that secondary responder is less likely to

298 survive an attack and needs to respond early (i.e., has a greater value for $t$ ). Of course, time

299 required to reach safety may also depend upon how many other individuals are simultaneously

300 fleeing from consumers, and thus, complex games may emerge (Gil et al. 2018). Future work

301 could test hypotheses pertaining to the relationship between PFL, primary vs. secondary 
302 detectors/responders, and population level mortality to explore the transmission of risk

303 information among group members and across species (Valone and Templeton 2002; Gil et al.

304 2018).

305 Plants, although single individuals, have multiple redundant parts and may respond more

306 like a group of closely related animals than a single genetically-unique individual (Karban et al.

307 2016). When attack and consumption are initiated, plants respond by inducing defenses and

308 reallocating resources to unattacked and less accessible parts (Schultz et al. 2013). Considering

309 the plant as a group and each component as an individual allows us to ask similar questions as

310 we do with animal groups. For example, do tissues with a higher PFL have a faster and stronger

311 response to herbivory (McKey 1974; Zangerl and Rutledge 1996)? Does the propagation of risk

312 signals occur more quickly through young plants with higher PFL than through mature

313 individuals?

314 Individuals within clonal groups likely have very different responses than individuals

315 within unrelated/partially related groups, given that consumption of a single individual is

316 unlikely to result in death of the entire colony or clonal group (Harvell 1990). They can thus

317 gather highly reliable information late in the interaction sequence to optimize their defense

318 (assuming risk information transfer among clonal individuals occurs). For example, the

319 consumption of some soldiers in clonal aphid colonies alters colony-level allocation to defense

320 without high fitness costs (Aoki and Kurosu 2004). As such, use of risk information and

321 defensive investment should occur at the colony level, not at the individual level. This line of

322 reasoning can be further extended to provide novel insights into defensive investment as a

323 function of colony size or age: young, small colonies of clonal or eusocial organisms should

324 respond strongly and early in the interaction sequence because of the greater cost of losing some 
325 individuals, whereas large, mature colonies should respond less strongly at the same point in the

326 interaction sequence. An additional, unappreciated suggestion that follows from our perspective

327 is that the potential to reduce the costs of activating unnecessary defenses may provide a

328 selective advantage to clonality and eusociality. Alternatively, sessile prey may provide an

329 interesting contrast as a single individual that is immobile. Future studies comparing anti-

330 consumptive responses between clonal groups (animals or plants), sessile prey, and plant

331 individuals may provide further insights into the commonalities of defensive investment.

332 Importantly, although we emphasize the role of PFL in information transfer pertaining to

333 the arrival of consumers/predators, PFL also affects the ability of organisms to gain information

334 required to re-emerge from a defended state (a key determinant of the cost of defensive

335 responses; Gil et al. 2018). For example, the fact that organisms with a high PFL often remain in

336 refuges long after predators have left (e.g., Sih 1992) likely influences their willingness to enter

337 such areas. Once defense is initiated plants remain in the defended state for long periods of time;

338 relaxation of this state appears less responsive to cues than the initial induction (Huntzinger et al.

339 2004). Further, organisms with low PFL may re-emerge more quickly from a defensive state and

340 actually facilitate other organisms leaving (Gil et al. 2018). Thus, information about the loss of

341 risk may transfer from individuals with a low PFL to individuals with a higher PFL.

342 Across both prey and plants, our perspective emphasizes how the PFL of one individual

343 (as well as the components of a more modular individual, e.g., plants) can alter the efficacy of

344 signaling to another, and how the PFL of the second can in turn alter the efficacy of its response.

345 A general prediction is that information about increased risk should move from higher- to lower-

346 PFL individuals, while the opposite may occur for information regarding decreased risk. Future

347 work should focus on how risk information moves throughout groups comprised of individuals 
348 of similar age/state relative to more heterogeneous groups. In general, our 'susceptibility

349 perspective' illustrates how the value of information depends critically upon the state of the

350 sender relative to the receiver, something the receiver may or may not be able to estimate

351 (Danchin et al. 2004).

352

353 The propagation of risk information through communities

354 We also expect that the rate at which risk information propagates through food webs and

355 across trophic levels (e.g., trait-mediated indirect interactions) may be determined by the relative

356 PFL of species within the system. A simple expectation is that responses to predators should be

357 stronger in systems characterized by prey with relatively higher PFL. For example, Chase (2003)

358 showed that in systems dominated by vulnerable snails (high PFL), predator effects were strong

359 and cascaded to plant resources; however, in systems dominated by relatively invulnerable

360 snails, predator effects were weak and did not cascade to plant resources. Our framework would

361 predict that the reduction in defensive investment by plant resources is likely to be higher in the

362 former system compared to the later. Further, if the timing of response determines the ultimate

363 rate of propagation through a food web, then behaviorally-mediated cascades would be most

364 rapid in systems of prey with relatively high PFL. In addition, information about the loss of risk

365 (i.e., when predators or herbivores leave an area) may be most rapidly transmitted in

366 communities with organisms that have relatively low PFL. Conversely, primary responding

367 species may be those with the greatest competitive ability for resources, while those that transmit

368 information about the loss of risk may be the poorest competitors (Gil et al. 2018). Considering

369 both the PFL and competitive ability of species within a community may provide unique insights

370 into how risk information is transmitted among species. 
Our perspective may also provide novel insights and predictions pertaining to the

372 magnitude of trophic interactions and the relative strength of top-down and bottom-up factors

373 influencing ecosystem dynamics. For example, because of their extreme vulnerability if attacked

374 (i.e., if attacked they are likely to die and thus a high PFL), rodents and other small mammals

375 respond very early in the interaction sequence (e.g., to moonlight), thereby significantly

376 decreasing their consumption of seeds and plants when there is little risk information (Orrock

377 and Fletcher 2014). Alternatively, large ungulates (with low PFL) may not alter their foraging

378 behavior until an attack is imminent (Middleton et al. 2013), and their foraging activities may be

379 more driven by spatial and temporal variation in food quantity and quality (but see Valeix et al.

380 2009; Tambling et al. 2015). Trophic interactions in systems dominated by organisms with

381 relatively high PFL may be more driven by top-down processes because of the risk-induced

382 shifts in herbivore behavior, while those dominated by organisms with relatively low PFL may

383 be more driven by bottom-up processes.

\section{Concluding remarks}

386 The obvious differences between prey and plants can lead us to overlook their

387 similarities: both live in variable environments with uncertain risk, and both utilize information

388 to maximize their fitness. Considering an organism's relative PFL if attacked and the influence

389 of PFL on the timing of information gathering and use across the interaction sequence provides a

390 common framework under which future studies can understand consumer-resource relationship.

391 Further, focusing on productive similarities between the disciplines will provide additional

392 insights and allow cross-talk of theories about the general consequences of consumptive

393 interactions. 
Although a variety of approaches may be used, comparative studies that use a range of

395 taxa and combinations of risk information cues will be particularly promising in helping

396 disentangle the role of PFL and information in affecting allocation to defense. As such, this

397 framework could be used to gain a better understanding of:

398 1) Why predation risk or herbivory seems more of a factor in some systems than others; for example, in systems where PFL is high (e.g., snowshoe hare - lynx) risk of consumption may be a greater factor than in systems where PFL is low (e.g., wolf-elk).

2) How ecological and environmental context influences consumer-resource interactions; for example: (a) Increases in resource (prey or plant) abundance may reduce PFL and thus alter responses to the risk of consumption; (b) The average toxicity of individuals in a population may alter consumer efficacy and thus alter PFL and resource responses to consumption; (c) In areas with more refuges individuals may have reduced PFL, because they can likely better escape when attacked, and thus, will delay their response to predation risk compared to areas without refuges; (d) Ambush predators may create a significant increase in PFL compared to cursorial predators, and this drives the earlier and greater response in prey (Schmitz 2007).

3) The role of consumer risk in mediating trophic interactions and how risk information transfers within and among systems; for example: (a) Understanding individual PFL may help distinguish between information transfer about impending/arriving risk vs. information transfer about safety (allowing organisms to resume activity); (b) Crossspecies information transfer may be mediated by the relative PFL of different species within the community.

4) How the ontogeny of prey and plants alters their investment in defense; for example, PFL 
likely differs across ontogeny and this may help predict changes in defensive investment, with stages where individuals have greater PFL having increased defensive investment.

\section{Acknowledgements}

421 We would like to thank Ken Schmidt for insightful comments on draft versions. JO gratefully

422 acknowledges support by the Wisconsin Alumni Research Foundation and the Vilas Mid-Career

423 Fellowship for a portion of this work. MJS was supported by a Fellowship from the Institute of

424 Advanced Studies, Hebrew University for a portion of this work. NSF IOS 1456724 to AS.

425 USDA NIFA 2018-67013-28068to JT. RK was supported by a fellowship from the Center for 426 Ecological Research, Kyoto University.

\section{References}

429 Agrawal AA, Laforsch C, Tollrian R (1999) Transgenerational induction of defenses in animals

$430 \quad$ and plants. Nature 401: 60-63

431 Aoki S, Kurosu U (2004) How many soldiers are optimal for an aphid colony? J Theoret Biol $432 \quad 230: 313-317$

433 Bateman AW, Vos M, Anholt BR (2014) When to defend: Antipredator defenses and the $434 \quad$ predation sequence. Am Nat 183:847-855

435 Bednekoff PA (1997) Mutualism among safe, selfish sentinels: a dynamic game. Am Nat $436 \quad 150: 373-390$.

437 Bednekoff PA, Lima SL (1998a) Randomness, chaos and confusion in the study of antipredator $438 \quad$ vigilance. Trends Ecol Evol 13:284-287 
439 Bednekoff PA, Lima SL (1998b) Re-examining safety in numbers: interactions between risk

440 dilution and collective detection depend upon predator targeting behaviour. Proc R Soc Lond

$441 \quad$ B $265: 2021-2026$

442 Brown JS, Kotler BP (2004) Hazardous duty pay and the foraging cost of predation. Ecology

443 Letters 10: 999-1014

444 Brown JS, Laundré JW, Gurung M (1999) The ecology of fear: Optimal foraging, game theory,

445 and trophic interactions. J Mammal 80:385-399

446 Caro T (2005) Antipredator Defenses in Birds and Mammals. University of Chicago Press,

$447 \quad$ Chicago.

448 Chase JM (2003) Experimental evidence for alternative stable equilibria in a benthic pond food

449 web. Ecol Lett 6: 733-741

450 Chittka L, Skorupski P, Raine NE (2009) Speed-accuracy tradeoffs in animal decision

451 making. Trends Ecol Evol 24:400-407

452 Clinchy M, Sheriff MJ, Zanette L (2013) Predator-induced stress and the ecology of fear. Funct

$453 \quad$ Ecol 27:56-65

454 Coley PD, Bryant JP, Chapin III FS (1985) Resource availability and plant antiherbivore

455 defense. Science 230:895-899

456 Danchin E, Giraldeau LA, Valone TJ, Wagner RH (2004) Public information: from nosy

457 neighbors to cultural evolution. Science 305:487-491

458 Donelan SC, Hellmann JK, Bell AM, Luttbeg B, Orrock JL, Sheriff MJ, Sih A (2020)

459 Transgenerational plasticity in human-altered environments. Trends Ecol Evo 35: 115-124

460 Falk KL, Kästner J, Bodenhausen N, Schramm K, Paetx C, Vassão DG, Reichelt M, von Knorre

461 D, Bergelson J, Erb M, Gershenzon J, Meldau S (2014) The role of glucosinolates and the 
462 jasmonic acid pathway in resistance of Arabidopsis thaliana against molluskan herbivores.

$463 \quad$ Mol Ecol 23: 1188-1203

464 Fornoni J (2011) Ecological and evolutionary implications of plant tolerance to herbivory. Funct $465 \quad$ Ecol 25:399-407

466 Gil MA, Hein AM, Spiegel O, Baskett ML, Sih A (2018) Social information-mediated

467 behavioral correlations drive population and community dynamics. Trends Ecol Evol 33:535$468 \quad 548$

469 Guiden PW, Bartel SL, Byer NW, Shipley AA, Orrock JL (2019) Predator-prey interactions in 470 the Anthropocene: reconciling multiple aspects of novelty. Trends Ecol Evol, In Press 471 Hairston NG, Smith FE, Slobodkin LB (1960) Community structure, population control, and $472 \quad$ competition. Am Nat 94: 421-425

473 Hamilton WD (1971) Geometry for the selfish herd. J Theor Biol 31:295-311

474 Harvell CD (1990) The ecology and evolution of inducible defenses. Quart Rev Bio 65:323-340

475 Heil M (2014) Herbivore-induced plant volatiles: targets, perception, and unanswered questions.

$476 \quad$ New Phytol 204:297-306

477 Heil M, Karban R (2010) Explaining evolution of plant communication by airborne signals.

$478 \quad$ Trends Ecol Evol 25:137-144

479 Helms AM, DeMoraes CM, Tröger A, Alborn HT, Francke W, Tooker JF, Mescher MC (2017)

480 Identification of an insect-produced olfactory cue that primes plant defenses. Nature Comm $481 \quad 8: 337$

482 Helms AM, Ray S, Matulis NL, Kuzemchak MC, Grisales W, Tooker JF, Ali JG (2019)

483 Chemical cues linked to risk: Cues from below-ground natural enemies enhance plant 
484

485

486

487

488

489

490

491

492

493

494

495

496

497

498

499

500

501

502

503

504

505

506

defences and influence herbivore behavior and performance. Funct Ecol https://doi.org/10.1111/1365-2435.13297

Hermann SL, Thaler JS (2014) Prey perception of predation risk: volatile chemical cues mediate non-consumptive effects of a predator on a herbivorous insect. Oecologia 176: 669-676

Hilker M, Meiner T (2006) Early herbivore alert: insect eggs induce plant defense. J Chem Ecol 32: $1379-1397$

Holeski L, Jander G, Agrawal AA (2012) Transgenerational defense induction and epigenetic inheritance in plants. Trends Ecol Evol 27: 618-626

Hunter MD (2016) The Phytochemical Landscape; Linking Trophic Interactions and Nutrient Dynamics. Princeton University Press, 376pp

Huntzinger M, Karban R, Young TP, Palmer TM (2004) Relaxation of induced indirect defenses of acacuas following exclusion of mammalian herbivores. Ecology 85: 609-614

Jensen EL, Dill LM, Cahill JF Jr. (2011) Applying behavioural-ecological theory to plant defense: light-dependent movement in Mimosa pudica suggests a trade-off between predation risk and energetic reward. Am Nat 177: 37-381

Karban R, Baldwin IT (1997) Induced Responses to Herbivory. University of Chicago Press, Chicago

Karban R, Agrawal AA, Thaler JS, Adler LS (1999) Induced plant responses and information content about risk of herbivory. Trends Ecol Evol 14:443-447

Karban R, Orrock JL, Preisser EL, Sih A (2016) A comparison of plants and animals in their responses to risk of consumption. Curr Opin Plant Biol 32:1-8

Kim J, Quaghebeur H, Felton GW (2011) Reiterative and interruptive signaling in induced plant resistance to chewing insects. Phytochemistry 72:1624-1634 
507 Lafferty KD, DeLeo G, Briggs CJ, Dobson AP, Gross T, Kuris AM (2015) A general consumer-

$508 \quad$ resource population model. Science 349:854-857

509 Lafferty KD, Kuris AM (2003) Trophic strategies, animal diversity and body size. Trends Ecol

$510 \quad$ Evol 17:507-513

511 Laundré JW, Hernández L, Altendorf KB (2001) Wolves, elk, and bison: reestablishing the

512 "landscape of fear" in Yellowstone National Park, USA. Can J Zool 79:1401-1409

513 Lima SL, Bednekoff PA (1999) Temporal variation in danger drives antipredator behavior: The

514 predation risk allocation hypothesis. Am Nat 153:649-659

515 Lima SL, Dill LM (1990) Behavioral decisions made under the risk of predation: a review and 516 prospectus. Can J Zool 68:619-640

517 Luttbeg B, Sih A (2010) Risk, resources and state-dependent adaptive behavioural syndromes.

518 Philos Trans R Soc Lond B Biol Sci 365: 3977-3990

519 Matassa CM, Donelan SC, Luttbeg B, Trussel GC (2016) Resource levels and prey state

520 influence antipredator behavior and the strength of nonconsumptive predator effects. Oikos

$521 \quad 125: 1478-1488$.

522 Markovic D, Colzi I, Taiti C, Scalone R, Ali JG, Mancuso S, Ninkovic V (2019) Airborne

523 signals synchronize the defenses of neighboring plants in response to touch. J Exp Bot 70:

$524 \quad 691-700$

525 McKey D (1974) Adaptive patterns in alkaloid physiology. Am Nat 108:305-320

526 McNamara JM, Houston AI (1986) The common currency for behavioral decisions. Am Nat

$527 \quad 127: 358-378$

528 McNamara JM, Houston AI (1992) Risk-sensitive foraging: A review of the theory. Bltn

$529 \quad$ Mathcal Biology 54: 355-378 
530 Middleton AD, Kauffman MJ, McWhirter DE, Jimenez MD, Cook RC, Cook JG, Albeke SE,

531 Sawyer H, White PJ (2013) Linking anti-predator behaviour to prey demography reveals

532 limited risk effects of an actively hunting large carnivore. Ecol Lett 16:1023-1030

533 Minorsky PV (2019) The functions of foliar nyctinasty: a review and hypothesis. Biological

$534 \quad$ Reviews 94: 216-229

535 Niu Y, Sun H, Stevens, M (2018) Plant camouflage: ecology, evolution, and implications.

$536 \quad$ Trends Ecol Evol 33:608-618

537 Ohgushi T (2005) Indirect interaction webs: Herbivore-induced effects through trait change in 538 plants. Ann Rev Ecol Evol Syst 36:81-105

539 Orrock JL (2013) Exposure of unwounded plants to chemical cues associated with herbivores

$540 \quad$ leads to exposure-dependent changes in subsequent herbivore attack. PLoS One 8:e79900

541 Orrock JL, Connolly BM, Choi WG, Guiden PW, Swanson SJ, Gilroy S (2018) Plants eavesdrop

542 on cues produced by snails and induce costly defenses that affect insect herbivores.

$543 \quad$ Oecologia 186: 703-710

544 Orrock JL, Fletcher Jr RJ (2014) An island-wide predator manipulation reveals immediate and

$545 \quad$ long-lasting matching of risk by prey. Proc R Soc B 281:20140391

546 Orrock JL, Sih A, Ferrari MCO, Karban R, Preisser EL, Sheriff MJ, Thaler JS (2015) Error

547 management in plant allocation to herbivore defense. Trends Ecol Evol 30:441-445

548 Parsons MH, Apfulback R, Banks PB, Cameron EZ, Dickman CR, Frank ASK, Jones ME,

549 McGregor IA, McLean S, Müller-Schwarze D, Sparrow EE, Blumstein DT (2018)

550 Biologically meaningful scents: a framework for understanding predator-prey research across

551 disciplines. Biological Reviews 93: 98-114 
552 Peiffer M, Tooker JF, Luthe DS, Felton GW (2009) Plants on early alert: glandular trichomes as

553 sensors for insect herbivores. New Phytol 184:644-656

554 Preisser EL, Orrock JL (2012) The allometry of fear: interspecific relationships between body $555 \quad$ size and response to predation risk. Ecosphere 3:77.

556 Prugh LR, Golden CD (2014) Does moonlight increase predation risk? Meta-analysis reveals

557 divergent responses of nocturnal mammals to lunar cycles. J Anim Ecol 83: 504-514

558 Raffel TR, Martin LB, Rohr JR (2008) Parasites as predators: unifying natural enemy ecology.

$559 \quad$ Trends Ecol Evol 23: 610-618

560 Reznick DA, Bryga H, Endler JA (1990) Experimentally induced life-history evolution in a

$561 \quad$ natural population. Nature 346:357-359

562 Reznick DA, Endler JA (1982) The impact of predation on life history evolution in Trinidadian 563 guppies (Poecilia reticulata). Evolution 36:160-177

564 Rossiter MC (1996) Incidence and consequences of inherited environmental effects. Ann Rev

$565 \quad$ Ecol System 27: 451-476

566 Schmitz OJ, Miller JRB, Trainor AM, Abrahms B (2017) Toward a community ecology of

567 landscapes: predicting multiple predator-prey interactions across geographic space. Ecology

$568 \quad 98: 2281-2292$

569 Schultz JC, Appel HM, Ferrieri AP, Arnold TM (2013) Flexible resource allocation during plant 570 defense responses. Front Plant Sci 4:1-11

571 Sheriff MJ, Bell A, Boonstra R, Dantzer B, Lavergne SG, McGhee KE, MacLeod KJ, Winandy

572 L, Zimmer C, Love OP (2017) Integrating ecological and evolutionary context in the study of 573 maternal stress. Int Comp Biol 57: 437-449 
574 Sheriff MJ, Krebs CJ, Boonstra R (2010) The ghosts of predators past: population cycles and the

575 role of maternal programming under fluctuating predation risk. Ecology 91:2983-2994

576 Sheriff MJ, Peacor S, Hawlena D, Thaker M (2020) Non-consumptive predator effects on prey

577 population size: a dearth of evidence. J Anim Ecol doi.org/10.1111/1365-2656.13213

578 Sih A (1992) Prey uncertainty and the balancing of antipredator behavior and feeding needs. Am

$579 \quad$ Nat 139:1052-1069

580 Sih A (2005) Predator-prey space use as an emergent outcome of a behavioral response race. In

581 Ecology of Predator-Prey Interactions pp. 240-255, Oxford University Press, USA

582 Smith JA, Donadio E, Pauli JN, Sheriff MJ, Middleton AD (2019) Integrating temporal refugia

583 into landscapes of fear: prey exploit predator downtimes to forage in risky places. Oecologia

$584 \quad$ https://doi.org/10.1007/s00442-019-04381-5

585 Song YY, Ye M, Li C, He X, Zhu-Salzman K, Wang RL, Su YJ, Luo SM, Zeng RS (2014)

586 Hijacking common mycorrhizal networks for herbivore-induced defence signal transfer

587 between tomato plants. Sci Reports 4: doi:10.1038/srep03915

588 Stamp N (2003) Out of the quagmire of plant defense hypotheses. Quart Rev Bio 78:23-55

589 Stankowich T, Blumstein DT (2005) Fear in animals: a meta-analysis and review of risk

$590 \quad$ assessment. Proc R Soc B 272:2627-2634

591 Stowe KA, Marquis RJ, Hochwender CG, Simms EL (2000) The evolutionary ecology of

592 tolerance to consumer damage. Annu Rev Ecol Evol Systemat 31:565-595

593 Strauss SY, Agrawal AA (1999) The ecology and evolution of plant tolerance to herbivory.

594 Trends Ecol Evol 14:179-185

595 Strong DR (1992) Are trophic cascades all wet? Differentiation and donor-control in speciose 596 ecosystems. Ecology 73:747-754 
597 Tambling CJ, Minnie L, Meyer J, Freeman EW, Santymire RM, Adendorff J, Kerley GI (2015)

598 Temporal shifts in activity of prey following large predator reintroductions. Behavioral Ecol

$599 \quad$ Sociobiol 69: 1153-1161.

600 Tigreros N, Norris R, Wang E, Thaler JS (2017) Maternally induced intraclutch cannibalism: an

$601 \quad$ adaptive response to predation risk? Ecol Lett 20: 487-494

602 Valeix M, Loveridge AJ, Chamaillé-Jammes S, Davidson Z, Murindagomo F, Fritz H,

603 Macdonald DW (2009) Behavioral adjustments of African herbivores to predation risk by

604 lions: spatiotemporal variations influence habitat use. Ecology 90: 23-30.

605 Valone TJ, Templeton JJ, (2002) Public information for the assessment of quality: a widespread

606 social phenomenon. Phil Trans R Soc Lond B 357: 1549-1557.

607 Weissburg MJ, Smee DL, Ferner MC (2014) The sensory ecology of nonconsumptive predator

$608 \quad$ effects. Am Nat 184:141-157

609 Werner JR, Krebs CJ, Donker SA, Sheriff MJ (2015) Forest or meadow: the consequences of

610 habitat for the condition of female arctic ground squirrels (Urocitellus parryii plesius). Can J

$611 \quad$ Zool 93: 791-797

612 Wishingrad V, Ferrari MCO, Chivers DP (2014) Behavioural and morphological defences in a

613 fish with a complex antipredator phenotype. Anim Behav 95: 137-143

614 Ydenberg RC, Dill LM (1986) The economics of fleeing from predators. Adv Study Behav 16:

$615 \quad 229-249$

616 Zangerl AR, Rutledge CE (1996) The probability of attack and patterns of constitutive and

617 induced defense: a test of optimal defense theory. Am Nat 147:599-608

618 


\section{Box. 1. Proportional fitness loss of an individual and its timing of defense}

620 We suggest the proportional fitness loss (PFL) if an individual does not initiate defense until

621 attacked is a critical component of understanding its defensive investment. PFL relies on an

622 individual's fitness potential if it initiates defense prior to an attack compared to its fitness

623 potential if it initiates defense during an attack. For example, if individual A is 50\% likely to

624 survive if it defends during the detection stage and only $20 \%$ likely to survive if it defends during

625 an attack, its PFL is $60 \%$ ((50-20)/50). If individual B has a 95\% chance of surviving if it

626 defends during the detection stage and a 30\% chance of survival if it defends during an attack, its

627 PFL is $68 \%$. From this scenario, it becomes clear that the PFL of an individual depends on both

628 its ability to survive an attack and, also, the effectiveness of its early defense. In such a scenario

629 individual B has a higher PFL and should initiate defense earlier than individual A, even though

630 it has a higher probability of surviving an attack. Our concept helps to clarify why individuals

631 with low expected fitness, regardless of whether they initiate defense early or late (thus a low

632 PFL), would be expected to wait and initiate defense late (if at all) given the ineffectiveness of

633 their (early) defense.

634 The fact that consumer-resource encounters progress through time along a common

635 interaction sequence of events (Lima and Dill 1990; Karban and Baldwin 1997, Caro 2005; Fig.

636 1) allows us to build relative PFL curves across the interaction sequence to better understand the

637 timing of defensive investment. As individuals delay their defensive investment their fitness

638 potential will approach that which they would have if they did not invest in defense until

639 attacked. This also allows us to visualize inflection points where fitness potential will greatly

640 decrease if defense is not initiated. In the first two examples above, the fitness potential

641 difference between early and late defensive investment is relatively large and, if their PFL curve 
642 was relatively linear, both individuals may greatly increase their survival for incremental

643 advances in the timing of their defense. Alternatively, if, for example, we extend the above

644 scenario such that individual A had a 95\% chance of survival if it defended during the encounter

645 stage (thus a PFL of $79 \%$ between encounter and attack, but a 47\% PFL between encounter and

646 detection), while individual B had a $99.9 \%$ chance of survival if it defended during the encounter

647 stage (thus a PFL of $70 \%$, but only 4\% PFL between encounter and detection), our curve would

648 predict that individual A would most benefit from defending during the encounter stage, while

649 individual B may benefit from delaying defensive investment until the detection stage.

650 Additionally, if individuals invest too early or respond to unreliable information they will

651 pay a cost of unnecessary defense (e.g., cost of defense itself, missed opportunity costs,

652 reductions in growth and reproduction). The willingness of individuals to pay a cost of

653 unnecessary defense will also depend on their PFL. Individuals with a high PFL can pay a

654 relatively high cost of unnecessary defense and still benefit significantly from early defensive

655 investment. Alternatively, individuals with a low PFL if attacked may not be willing to pay as

656 high a cost of unnecessary defense and should defend relatively later.

657 While we discuss the fitness aspect of PFL as a loss of survival, individual fitness could

658 also be measured as a loss of reproduction (number of babies born or weaned, loss of litters, loss

659 of seed set or flowers, etc.) or a loss of growth or tissue (in many species growth is directly

660 related to reproductive potential and in the case of plants or other organisms that can be partially

661 consumed a loss of tissue may be a better metric) if attacked. It is important to appreciate that in

662 these latter two fitness measures, with respect to PFL, the loss of fitness is due to attack not the

663 initiation of defense (as is often the case). Because of this, however, these latter two fitness

664 measures may be particularly insightful given i) they can be used to estimate PFL if attacked but 
665 also the cost of unnecessary defense (defending too early) and ii) that they can be used to

666 estimate the loss of relative fitness at any point along the interaction sequence when defense is

667 initiated. Understanding an individual's PFL across the interaction sequence will provide

668 valuable insights into when it should initiate defense and has significant implications for

669 understanding how prey and plants will respond to the risk of consumption.

670 
671 Figure 1. An individual's proportional fitness loss if defense is initiated during attack (PFL; Box

672 1) should reflect the timing and magnitude of defensive investment across the interaction

673 sequence. We highlight two qualitative scenarios that represent the range of possibilities we

674 envision. (Dashed Line) Individuals that experience rapid, significant losses of fitness once an

675 attack begins (i.e., individuals with higher PFL; dashed line panel A) should implement defenses

676 relatively early in the interaction sequence (dashed line panel B), as implementation of early

677 defenses maximizes that likelihood that the predator or herbivore attack will be unsuccessful or

678 attack will not occur. (Solid Line) Individuals that experience lower PFL if an attack begins, and

679 may even be able to survive partial consumption (solid line panel A), should respond late in the

680 encounter sequence (solid line panel B) in order to minimize the costs of unnecessary defense.

681 The magnitude of defense exhibited by individuals during the pre-encounter stage may depend

682 upon their prior experience, as such individuals with higher PFL (dashed line) will likely have

683 higher defensive investment during this stage. While individuals can alter the sequence by

684 escaping during this stage (and thus entering the escape recovery stage directly), the return of

685 individuals to a baseline defense level (panel B) and maximum fitness potential (panel A) will

686 likely be slower in individuals with higher PFL; which in turn will alter the initial magnitude of

687 defense during the pre-encounter stage. Because this is a relative scale, the magnitude and timing

688 of defensive investment may differ among individuals within and among populations or among

689 individuals of different species within a community. Note that as the interaction sequence

690 progresses the reliability of risk information also increases.

691 
692 Fig. 1
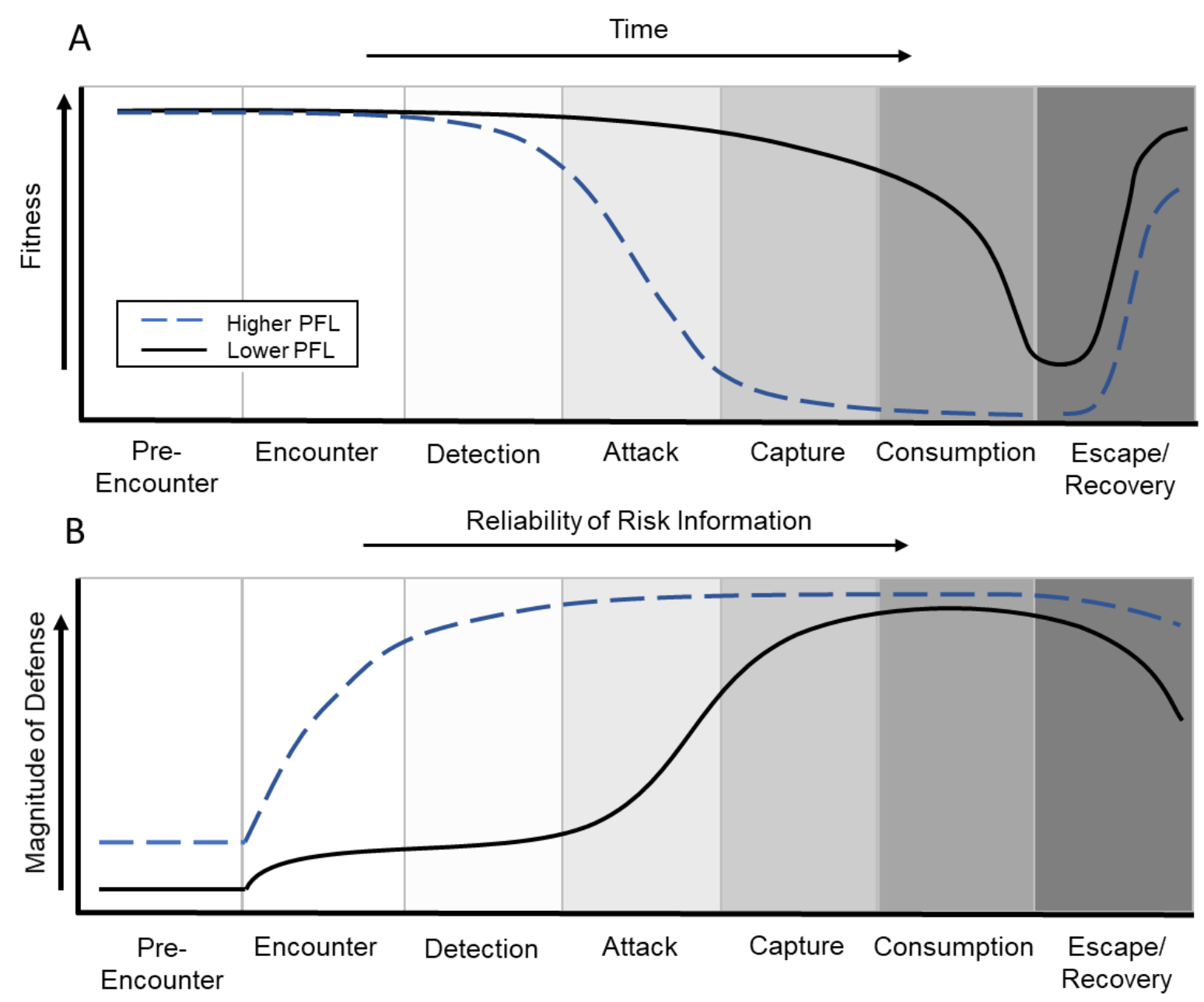

693

694

695 
696 Fig. 2. The common sequence of attacked-related events shared during both predator-prey and

697 herbivore-plant interactions. Interactions begin during a pre-encounter spatiotemporal overlap

698 stage and end in a post-encounter escape/recovery stage (or death if consumption is lethal).

699 Depending upon the timing and magnitude of prey/plant defense (which is reliant on their PFL),

700 prey/plants can avoid, deter, and escape from their consumers altering the outcome of this

701 sequence at any stage and enter the post-encounter stage (dashed lines). Importantly, this

702 sequence does not proceed in a simple linear fashion and any previous encounter with a

703 consumer will influence future encounters (i.e., the post-encounter experience will influence pre-

704 encounter defensive investment).

705

706 Fig. 2.

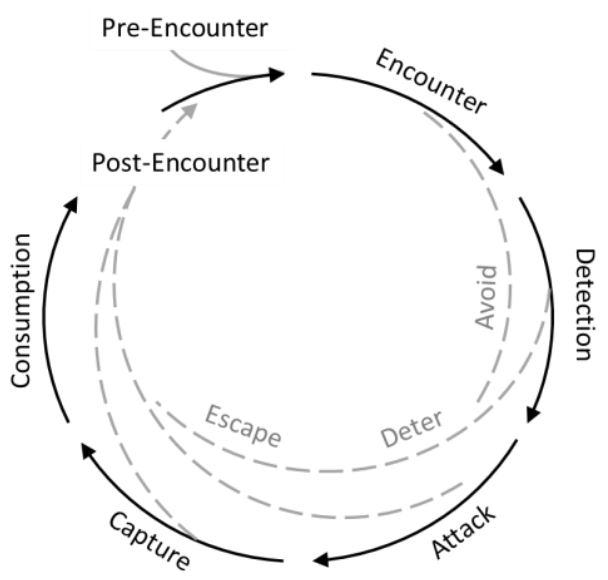

